\title{
Topological Insulator State and Collapse of the Quantum Hall Effect in a Three-Dimensional Dirac Semimetal Heterojunction
}

\author{
David A. Kealhofer $\odot,{ }^{1}$ Luca Galletti $\odot,{ }^{2}$ Timo Schumann $\odot,{ }^{2}$ Alexey Suslov $\odot,{ }^{3}$ and Susanne Stemmer ${ }^{2, *}$ \\ ${ }^{1}$ Department of Physics, University of California, Santa Barbara, California 93106-9530, USA \\ ${ }^{2}$ Materials Department, University of California, Santa Barbara, California 93106-5050, USA \\ ${ }^{3}$ National High Magnetic Field Laboratory, Tallahassee, Florida 32310, USA
}

(Received 10 July 2019; revised manuscript received 25 November 2019; accepted 23 December 2019; published 27 February 2020)

\begin{abstract}
Thin films promise new opportunities for the manipulation of surface states of topological semimetals with the potential to realize new states that cannot be obtained in bulk materials. Here, we report transport studies of gated Hall bar structures fabricated from approximately 50-nm-thick, (001)-oriented epitaxial films of cadmium arsenide, a prototype three-dimensional Dirac semimetal, in magnetic fields up to $45 \mathrm{~T}$. The films exhibit a quantized Hall effect with pronounced odd-integer plateaus that is strikingly different from that of the more widely studied (112)-oriented films. We show that the unusual quantum Hall effect is a consequence of the inverted bulk band structure of cadmium arsenide that creates topological-insulatorlike states at the bottom and top interfaces, each exhibiting a half-integer quantum Hall effect. A small potential offset between the two surfaces results in the crossing of the Landau levels and gives rise to the filling factor sequences observed in the experiments. Moreover, at large negative values of gate bias, the filling factor $\nu=1$ is abruptly preempted by an insulating state that is accompanied by the collapse of the well-developed quantum Hall effect. We suggest that this new phase cannot be explained within a singleparticle picture and discuss the role of Coulomb interactions between spatially separated surface states.

DOI: $10.1103 /$ PhysRevX.10.011050

Subject Areas: Condensed Matter Physics,

Topological Insulators
\end{abstract}

\section{INTRODUCTION}

Gapless boundary states are a key signature of gapped topological phases, such as three-dimensional topological insulators (3D TIs) and quantum spin Hall insulators, which are of great interest for applications ranging from spintronics to quantum computing [1-3]. In recent years, 3D Dirac semimetals - materials that host doubly degenerate nodes around which the single-particle dispersion can be described by a version of the Dirac equation-have broadened the family of topological materials beyond these gapped phases [4]. Such semimetals host Fermi arcs on surfaces for which the bulk nodes project onto separate points in the surface Brillouin zone, which may form a "Weyl orbit" for quasiparticles, connecting both surfaces through the bulk nodes [5-7]. In addition, topological semimetals can host Dirac surface states that resemble those of 3D TIs [8-10]. In contrast to Fermi arcs, these are

\footnotetext{
* Corresponding author. stemmer@mrl.ucsb.edu

Published by the American Physical Society under the terms of the Creative Commons Attribution 4.0 International license. Further distribution of this work must maintain attribution to the author(s) and the published article's title, journal citation, and DOI.
}

largely unstudied in 3D Dirac semimetals, though some of the theoretical framework was laid long ago $[11,12]$. In high-quality semimetal films, which have recently become available and in which bulk transport can be minimized [13-15], it is possible to isolate these surface states and reveal physics that is generally obscured by bulk parasitic effects in first-generation 3D TIs. These surface states are expected to give rise to unique magnetoelectric phenomena, including a half-integer quantum Hall effect [3]. Looking further, nearly every experimental observation of topological surface states to date has been understood within a framework of noninteracting quasiparticles. Realizing novel phases that arise from a combination of Coulomb interactions, strong spin-orbit coupling, and topology is a highly sought-after goal in the field of topological matter $[16,17]$.

In this paper, we investigate the quantum Hall effect of thin, (001)-oriented cadmium arsenide $\left(\mathrm{Cd}_{3} \mathrm{As}_{2}\right)$, a prototype 3D Dirac semimetal [8,18-22]. This combination of material, crystal orientation, and sample geometry is significant, because the two Dirac nodes lie along the $k_{z}$ axis and project onto the same point in the (001) surface Brillouin zone $[8,23]$. As a result, bulk and surface states coincide unless a gap opens in the bulk, due to, e.g., confinement, structural inversion asymmetry [24,25], or epitaxial strain. In these films, the thickness (approximately $50 \mathrm{~nm}$ ) is great enough that a hybridization gap does not 


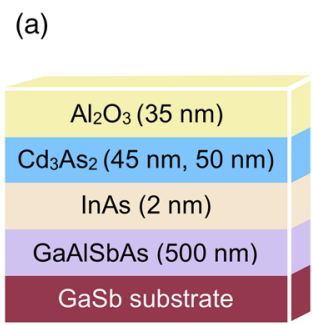

(b)
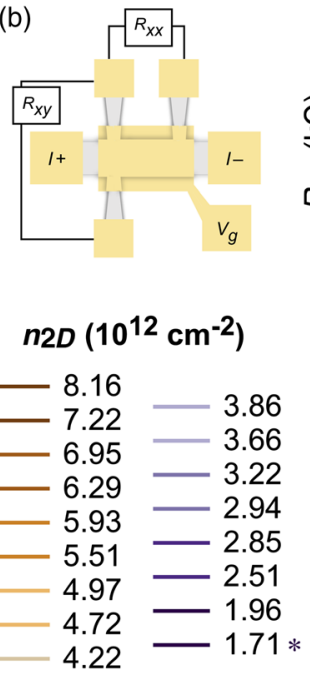

(c)

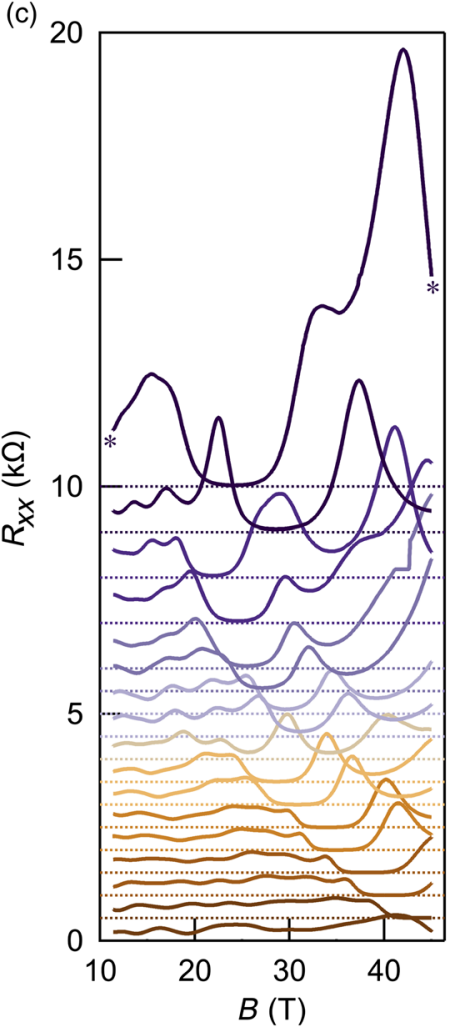

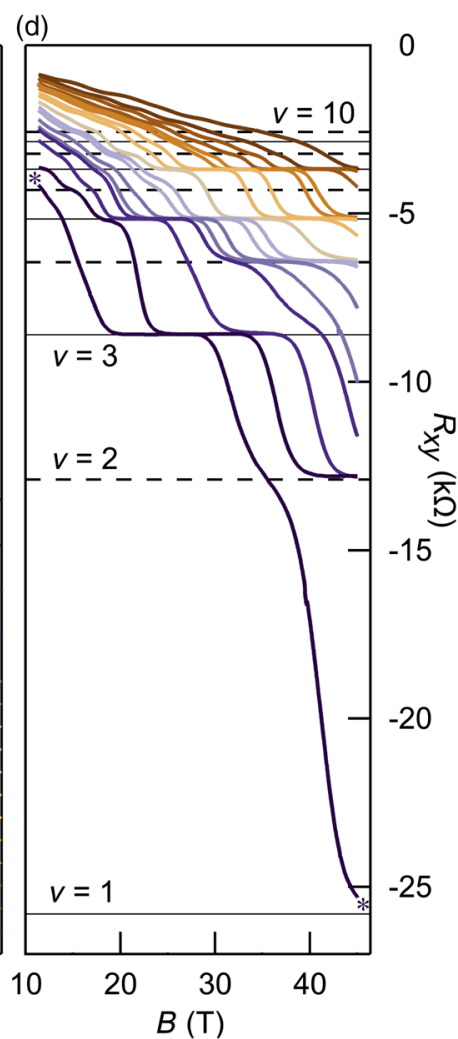

FIG. 1. (a) Schematic of heterostructure (not to scale). (b) Schematic of the gated Hall bar device (not to scale). The gate metal is separated from the mesa by the $\mathrm{Al}_{2} \mathrm{O}_{3}$ layer, and the other contacts are in contact with the $\mathrm{Cd}_{3} \mathrm{As}_{2}$ layer. Note that the contacts for current injection and voltage measurement connect both $\mathrm{Cd}_{3} \mathrm{As}_{2}$ layer surfaces. (c) Longitudinal magnetoresistance $\left(R_{x x}\right)$ and (d) Hall resistance $\left(R_{x y}\right)$ for the device fabricated on a 45-nm-thick $\mathrm{Cd}_{3} \mathrm{As}_{2}$ layer. Different traces correspond to different gate biases or, equivalently, carrier densities. The 2D carrier densities are calculated from the 11.5 to $11.75 \mathrm{~T}$ Hall resistance and correspond to gate bias from about +3 to $-4 \mathrm{~V}$. In (c), traces are offset for legibility. For each trace, zero is indicated by a dotted horizontal line. In (d), odd (even) filling factors are indicated by solid (dashed) lines.

open in the surface states. We show that the quantum Hall effect is quantitatively different from that of the more widely studied (112) surface orientation [13-15,26]. The Landau level spectrum is consistent with topologicalinsulator-like surface states and cannot be described as originating from the subband states of a quantum well. Furthermore, and most strikingly, the filling factor $\nu=1$ is preempted by an insulating state. We suggest that this state, alongside other salient features in the quantum Hall data, requires an interpretation beyond a single-particle picture.

\section{EXPERIMENTAL}

(001)-oriented $\mathrm{Cd}_{3} \mathrm{As}_{2}$ thin films are grown by molecular beam epitaxy on a compound semiconductor structure, which is shown schematically in Fig. 1(a). As described in more detail elsewhere [27], the structure consists of a doped GaSb substrate, covered by an insulating GaAlSbAs buffer layer, a thin InAs wetting layer, and $\mathrm{Cd}_{3} \mathrm{As}_{2}$. The thicknesses of the two $\mathrm{Cd}_{3} \mathrm{As}_{2}$ films investigated here are 45 and $50 \mathrm{~nm}$, as confirmed by x-ray reflectivity. For the gated Hall bars [Fig. 1(b)], mesas are isolated by $\mathrm{Ar}$ ion milling, and the $\mathrm{Al}_{2} \mathrm{O}_{3}$ gate dielectric is deposited using a lowtemperature $\left(120^{\circ} \mathrm{C}\right)$ atomic layer deposition process. Ohmic and gate contacts $(\mathrm{Au} / \mathrm{Pt} / \mathrm{Ti})$ are deposited by electron-beam deposition. Magnetoresistance measurements are performed at the $45 \mathrm{~T}$ hybrid magnet at the National High Magnetic Field Laboratory, using the $300 \mathrm{mK}{ }^{3} \mathrm{He}$ insert. The resistance data are acquired using standard low-frequency lock-in techniques and subjected to a moving average smoothing routine before plotting.

\section{RESULTS}

Figures 1(c) and 1(d) show the longitudinal resistance $R_{x x}$ and Hall resistance $R_{x y}$ for the $45-\mathrm{nm}$-thick film as a function of the magnetic field under different values of top gate bias, corresponding to a variation in the total $2 \mathrm{D}$ carrier density ranging from $8.2 \times 10^{12}$ to $1.7 \times 10^{12} \mathrm{~cm}^{-2}$. The corresponding plots for the 50-nm-thick film are shown in Supplemental Material [28]. An unusual feature of the quantum Hall effect observed here is the prominence of plateaus with odd filling factors $\nu$ [indicated by the solid 

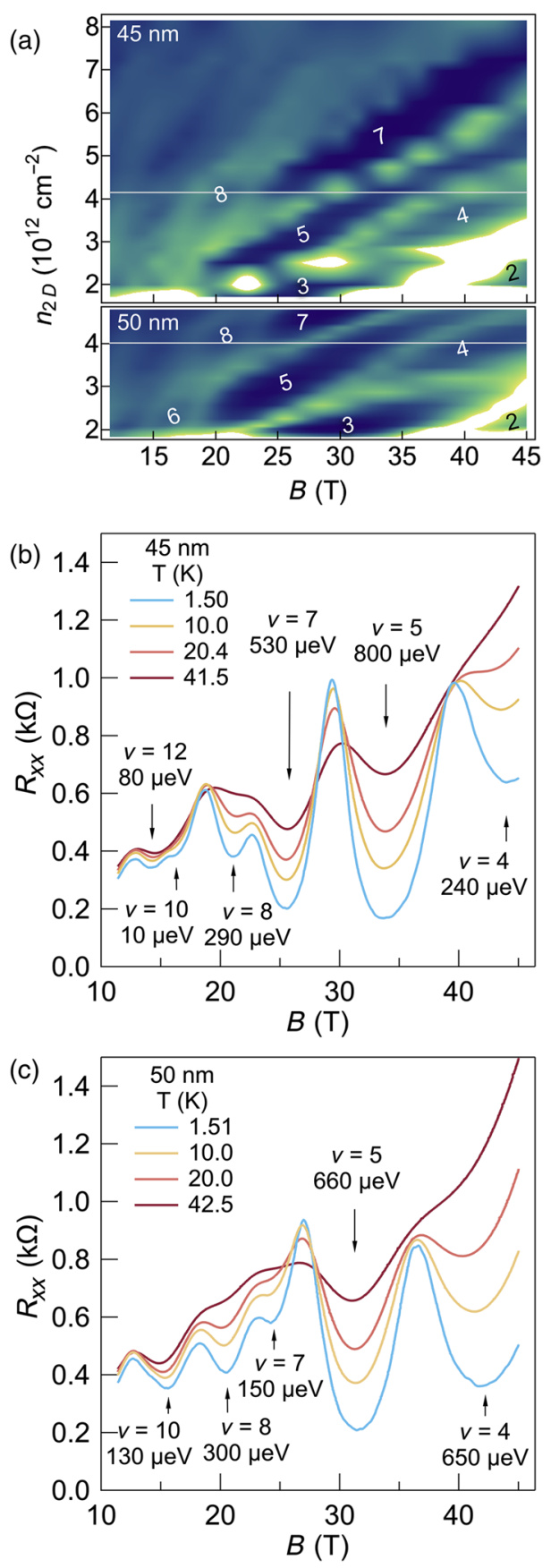

FIG. 2. (a) Maps of the longitudinal magnetoresistance (scaled from dark blue to light green, indicating low to high) for devices made from the 45- to 50-nm films, respectively, as a function of the magnetic field and carrier density. The two panels have the same vertical and horizontal scales. Thin horizontal gray lines correspond to the carrier density for the traces in (b) and (c). The spotty features are artifacts of the 2D plotting procedure. These are the same data as shown in Figs. 1(c) and 1(d) and Supplemental Figs. S1(a) and S1(b) [28]. (b) Temperature-dependent Shubnikov-de Haas oscillations from 1.5 to $42 \mathrm{~K}$ for the 45 -nm-thick sample. The activation energies shown are calculated from an Arrhenius-type fit to the indicated $R_{x x}$ minima, labeled by the filling factor deduced from the corresponding value of $R_{x y}$. These traces would fall along the thin gray horizontal line on the 2D map in Fig. 2(a). (c) The same as (b) from 1.5 to $43 \mathrm{~K}$ for the 50 -nm-thick sample. lines in Fig. 1(d)]. Independent of the carrier density, the quantization of $R_{x y}=(1 / \nu)\left(h / e^{2}\right)$ is more exact, and the plateaus more fully developed, for $\nu=3,5$, and 7. By contrast, plateaus occurring at even filling factors (dashed lines) in many cases do not develop fully: There are plateaulike features in the Hall resistance, but the slope $d R_{x y} / d B$ does not reach zero. This difference is also reflected in $R_{x x}$, where the minima approach zero more closely as the Hall resistance reaches plateaus at odd filling factors than at even ones.

The Landau level structure is also apparent in the maps of the longitudinal resistance $R_{x x}$, plotted as a function of the carrier density and magnetic field, shown for both samples in Fig. 2(a). The behavior of the two samples is nearly identical. Regions with the same filling factor, determined by their $R_{x y}$ values, are oblong in shape and obey a complicated ordering scheme. For example, the filling factor increases by three between the regions $\nu=5$ and $\nu=8$; across the entire diagram, adjacent minima of $R_{x x}$ coincide with steps in the filling factor of 1,2 , and 3 . That the steps of 1 and 2 are not restricted to the high- or low-field regime indicates that these patterns are not due to the resolution of a degeneracy such as spin splitting but rather due to the overlap of more than one fan of Landau levels, each originating from different zero-field energy states.

As shown in Figs. 2(b) and 2(c), the lowest values of $R_{x x}$ coincide with the highest activation energies, deduced from an Arrhenius-type fit of the $T>1.5 \mathrm{~K}$ temperature dependence of the $R_{x x}$ minima, while higher $R_{x x}$ minima correspond to lower activation energies. In particular, the activation energy is greater at higher odd values of $\nu$ than lower even ones, in contrast to most conventional twodimensional systems for which the activation energy increases with decreasing $\nu$. [Apparent deviations in the temperature plots are due to where the plateau regions are traversed; see the white lines in Fig. 2(a).]

Figure 3 shows the behavior of the devices under a larger negative gate bias than shown in Fig. 1, with the darkest purple trace common to both figures (as indicated by the asterisks). This trace still has a well-developed $\nu=3$ plateau and approaches $\nu=1$. Under an increasingly negative bias, with $n_{2 D}<1.7 \times 10^{12} \mathrm{~cm}^{-2}$, however, a striking and abrupt change appears in the Hall resistance: it becomes nonlinear in magnetic field and ceases to form clear plateaus. Most tellingly, this behavior is not restricted to a particular magnetic field or carrier density (gate bias) alone but instead coincides with the combined parameters for which the plateau at $\nu=1$ is expected to develop fully. For some gate voltages, the quantum Hall plateaus vanish altogether. At the same time, the longitudinal resistance increases by more than a factor of 10 . Nevertheless, features in the longitudinal magnetoresistance depend on both the magnetic field and the carrier density. The behavior is similar for both films (see Fig. S1 in 

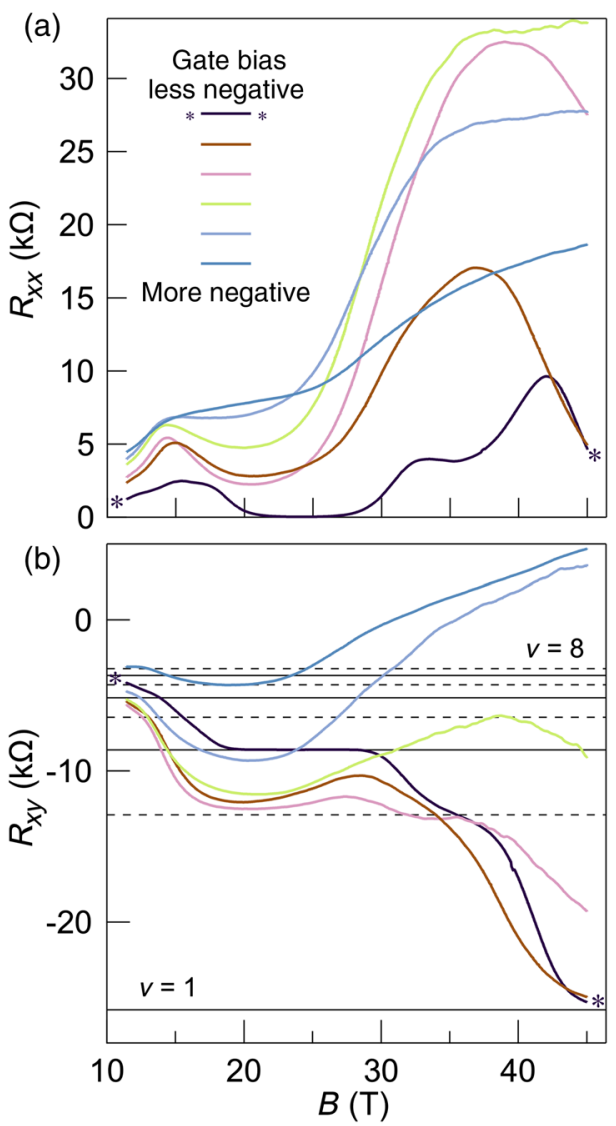

FIG. 3. (a) $R_{x x}$ and (b) $R_{x y}$ data under large negative bias ranging from -4 to $-11 \mathrm{~V}$. The dark purple traces marked with asterisks are carried over from Figs. 1(c) and 1(d) for comparison. In (b), odd (even) filling factors are indicated by solid (dashed) lines.

Supplemental Material [28]) and reproducible in successive field sweeps at different values of gate bias.

\section{DISCUSSION}

We next discuss the results, beginning with the observed Landau level sequences. The exact quantization of the Hall resistance and the concomitant near vanishing of the longitudinal magnetoresistance $(<10 \Omega$ in the widest plateaus) is strong evidence for the two-dimensional nature of the transport. In such relatively thick films, a purely twodimensional nature of the transport is strong evidence that surface states are responsible for the observed spectrum. As discussed below, these surface states are a consequence of the inverted band structure of the $\mathrm{Cd}_{3} \mathrm{As}_{2}$ bulk that is in contact with the conventionally ordered bands of the III-V semiconductor layer and gate dielectric on either side of the quantum well. As in the surface states of a 3D TI, this heterojunction should give rise to a half-quantized Hall effect on each surface, and only odd integer filling factors should be observed in the absence of electron-electron interactions and/or a potential difference across the bulk
[29-35]. Here, we show that the sequence of Landau levels observed in Figs. 1 and 2 is consistent with the helical 3D TI-like surface states. Figure 4(a) shows the Landau level spectra for the top (purple lines) and bottom (orange lines) surface states of a generic 3D TI [36] (see Supplemental Material for details of the model [28]). In Fig. 4(a), we take into account that the two surfaces, each hosting a massless surface state, differ by a small offset in potential energy due to differences between the bottom and top surface inherent to the heterostructure. Furthermore, a multiplicative factor heuristically accounts for the screening by the top surface in the action of the gate bias on the bottom one, which

(a)

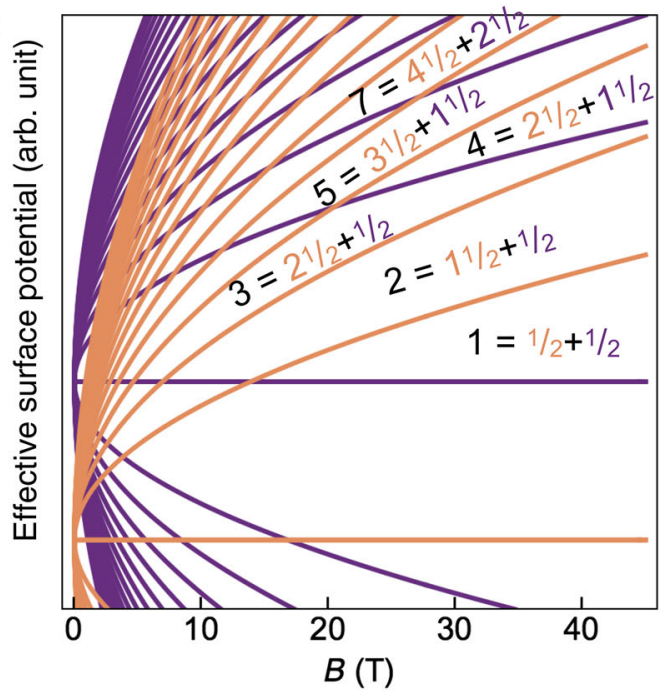

(b)
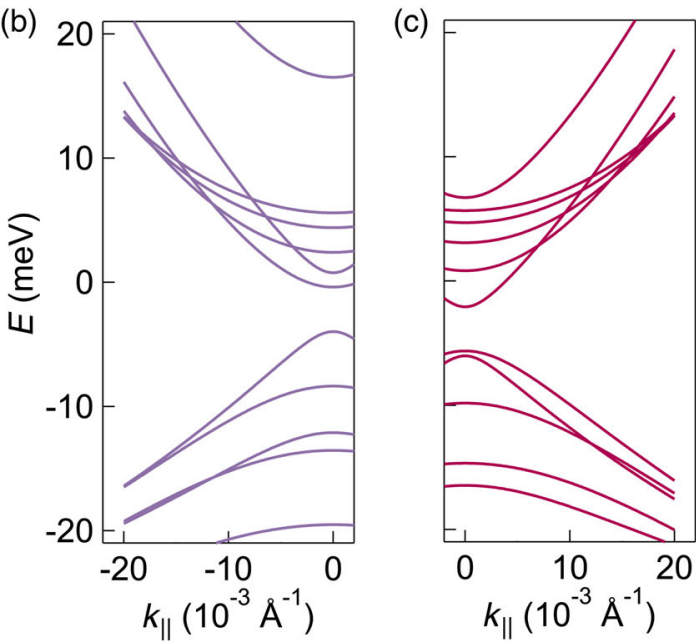

FIG. 4. (a) Schematic of Landau levels formed by two topologicalinsulator-like surface states, with filling factors labeled. The energy for two sets of Landau levels is plotted, with the energy of the set originating from the bottom surface scaled relative to the set originating from the top to account for the screening of the gate potential by the top surface. (b) Quantum well subbands formed in a 45 -nm-thick $\mathrm{Cd}_{3} \mathrm{As}_{2}$ film in a simple particle-ina-box picture. (c) Quantum well subbands formed in a 50-nmthick film. For details of the calculations, see Supplemental Material [28]. 
effectively modifies the potential difference as a function of the gate voltage. This scenario (in which the 2D carrier density is split between the two surfaces) fully explains the crossings of the Landau levels originating from each surface. Moreover, it reproduces the sequence of filling factors seen in both samples. Each zeroth Landau level, whose energy does not depend on the magnetic field, contributes a filling factor offset of $\frac{1}{2}$. Thus, we start counting from $\nu=1$ above the higher of the two zeroth Landau levels. Because of the single degeneracy of the massless states, the filling factor increases by one as we trace an upward path across a Landau level. The crossings of the Landau levels originating on each surface produce a sequence of odd integer filling factors in the intermediate field (approximately 20-30 T) range, matching the experimental data. The apparent jump of three in the filling factor from $\nu=5$ to $\nu=8$ is due to the close spacing of the $\nu=6$ and $\nu=8$ regions of the map. Finally, as a check of the selfconsistency of the Landau level assignments to each surface, we confirm that the carrier densities calculated from the top- and bottom-surface fan diagrams sum to those calculated from the Hall resistance for each gate bias (see Fig. S5 in Supplemental Material [28]).

We next briefly discuss the applicability of the topological surface state model for heterojunctions with (001) $\mathrm{Cd}_{3} \mathrm{As}_{2}$ films. Topological insulator surface states are intrinsic to heterojunctions formed at the interfaces between a film with inverted bands and "normal" insulators or semiconductors $[11,12,36]$. The bands in $\mathrm{Cd}_{3} \mathrm{As}_{2}$ are inverted in the sense that the " $S$ " band [see Eq. (S5) [28] ] is the lowest-lying state at $\Gamma$, while in the zinc-blende III-V compound the $S$ band is the conduction band. In $a b$ initio studies, the $S$ band is identified as a Cd $5 s$-like band that lies below the As $4 p$-like bands $[8,23]$. The difference in the crystal structure in each layer is captured simply, at the level of a $k \cdot p$ model, by a tetragonal distortion term that, in the absence of spin-orbit coupling, splits the degeneracy at $\Gamma$ of the states labeled $X Y Z$ in Eq. (S5) [28]. The tetragonal distortion term is responsible for the Dirac nodes at the Fermi level, and it sets the energy scale for the bulk Dirac physics $[8,37]$. At the interface, the $k \cdot p$ coefficients are interpolated between the values in each layer. The main effect is that crossings form due to the resolution of the band inversion $[11,12,36]$. This crossing produces exactly the chiral topological insulator state ("massless VolkovPankratov state") studied here.

Realistic $k \cdot p$ models for a thin $\mathrm{Cd}_{3} \mathrm{As}_{2}$ film sandwiched between InAs/GaAlSbAs and amorphous $\mathrm{Al}_{2} \mathrm{O}_{3}$ are expected to be more complicated; nevertheless, they should resolve the band inversion and, thus, result in a topological heterojunction. The model that forms the basis for the calculations shown in Fig. 4(a) uses a simple form of the topological heterojunction [36], as described in Supplemental Material [28]. Nevertheless, it reproduces the unusual filling factor sequence of the experimental data
[Fig. 2(a)]. A key difference between the two interfaces is the band offsets: the band crossings cannot be expected to be at the same energy. The model we use to describe the data is accordingly due to two topological heterojunctions, where the crossings are formed at different energies. As discussed above, this model captures the measured filling factor sequences and the structure of crossed Landau levels due to the different gate response of each surface. Furthermore, it suggests an energy scale for the visibility of the surface state in the hundreds of $\mathrm{meV}$, that is, the size of the band inversion, comparable to the high-field cyclotron energies. It is also consistent with the sensitivity of the quantum transport in these (001) films to the chemical condition of the top interface [27].

We next exclude other explanations for the observed filling factor sequences. We first emphasize that the observed sequences of Landau levels cannot originate from bulklike subbands formed by confinement in approximately 50-nm-thick quantum wells. Consider now the bands that form the 3D Dirac nodes. Using a four-band $k \cdot p$ model (for simplicity, we exclude the surface states; see also Ref. [28]) with the model parameters chosen to match first-principle calculations [38], we estimate the subband spacing to be less than $5 \mathrm{meV}$ for both films [see Figs. 4(b) and 4(c)]. For comparison, the Zeeman energy increases as at least $15 \mathrm{meV} / \mathrm{T}$, and the cyclotron energy varies as approximately $4 \mathrm{meV} / \mathrm{T}$. From this consideration, it seems impossible to see filling factors ranging from $\nu=1$ to $\nu=10$ in a sequence comparable to the data. A more detailed analysis confirms this (see Supplemental Material [28]). Furthermore, the confinement gap is a strongly varying function of the film thickness, and the subbands that form the gap change character three times every $10 \mathrm{~nm}$ [see Fig. S3(a) in Supplemental Material [28]]. As a result, the conduction and valence subband in-plane masses oscillate with the well thickness, even with relatively small thickness changes, as can be seen from Figs. 4(b) and 4(c). Thus, we would not expect the same sequence of filling factors in the two devices [cf. Figs. S3(b) and S3(c) in Supplemental Material [28] ].

Furthermore, the confinement gap depends nonmonotonically on the well thickness (see Supplemental Material [28]), and we would not expect to see the nearly identical spectra we see in the two devices [see Fig. 2(a)]. Moreover, the in-plane masses of the subbands forming the gap depend sensitively on the well thickness, even for the 45- and 50-nm-thick wells shown in Figs. 4(b) and 4(c). In contrast, signatures of the surface states are expected to be nearly independent of the film thickness, as long as it is large enough to prevent hybridization, which is consistent with these data. We note that there are reasons to expect a bulk gap in these films that is larger than in Fig. 4, for example, due to a small residual film strain [27] or structural inversion asymmetry [28], but these do not substantially alter the subband spacings. 
We can also exclude that the observed filling factors are due to competing Zeeman or cyclotron motion effects, i.e., originating from a zero-field spin degeneracy of a conventional two-dimensional electron gas. In particular, we note that the filling sequence at a constant magnetic field (or indeed at a constant carrier density) never occurs in steps of just two, as is a feature of such systems, whether with a low or high $g$ factor. Thus, the only other scenario is one in which two fans originating from a conventional, parabolic band have more or less evenly spaced Landau levels and extremely different masses. In such a case, to match the data, the lower fan must have an essentially nondispersive lowest Landau level, and the top fan must not. Moreover, in each fan, the $g$ factor must then change with the mass, so that the fans are approximately evenly spaced - this change is necessary to reproduce the observed steps of just one. This scenario is clearly unrealistic for $\mathrm{Cd}_{3} \mathrm{As}_{2}$, for which $g$ is measured to be approximately 20 , and the effective mass is found to be $0.03 m_{e}[8,19,22,39]$.

While topological-insulator-like surface states naturally explain the observed Landau level sequence, there are three features of the data that are not well captured by this singleparticle picture: (i) the observed difference in activation energy between the states at even and odd filling factorswhile specific sequences of odd vs even plateaus result from the two surfaces and their potential offsets with gate voltage, the picture underlying Fig. 4 does not result in a strong preference of odd vs even plateaus, in contrast to their systematically different activation energies in the experiments; (ii) the absence of a plateau at filling factor $\nu=1$ preceding (iii) the highly resistive behavior and breakdown of the quantum Hall effect under large negative bias. This behavior is all the more surprising, because from Fig. 4(a) we see that a total filling factor of $\nu=1$ corresponds to both surfaces being in their zeroth Landau level, or $\nu_{B}=\nu_{T}=1 / 2$, where the subscripts refer to bottom and top surfaces, respectively. These Landau levels are widely separated from other levels, and the $\nu=1$ plateau should be easily resolved under gate bias.

These observations can be explained by intersurface Coulomb interactions that depart from this single-particle picture. In particular, spatially separated Landau levels of the two surfaces are conceptually similar to the situation in bilayer two-dimensional electron gases of conventional III-V systems [40-42]. Such systems support a spontaneously coherent phase at $\nu_{B}=\nu_{T}=1 / 2$, which is described as a quantum Hall superfluid or exciton condensate. The collapse of the quantum Hall effect just before $\nu=1$ is reached and the highly resistive state observed here resemble the formation of such a condensate. In 3D TIs, correlation effects can give rise to minima in $R_{x x}$ at even filling factors, which have $\nu_{B} \neq \nu_{T}$ [41], and it would be interesting to understand if they can explain the observed difference in activation energies of even and odd plateaus. The ratio between intersurface separation and magnetic length is larger than in GaAs double quantum wells that exhibit exciton condensation [40]. We note, however, that a correlated $\nu=+1 / 2$ states is observed for large ratios in GaAs double quantum wells [43]. Furthermore, the spatial extent of the surface states is unknown.

Another explanation for the development of the insulating state before the plateau at $\nu=1$ develops is suggested by the surface state model shown in Fig. 4(a). In that picture, the total filling factor $\nu=1$ is formed by top and bottom filling factors $\nu_{T}=\nu_{B}=\frac{1}{2}$. In the presence of disorder, the mobile, extended states in the center of the Landau level may be few enough that, together with the fact that the top gate tunes the carrier density on each surface at different rates, it is impossible to tune the carrier density in such a way that the top and bottom surfaces are both close enough to half filling that the quantum Hall effect can still be observed. Instead, the top surface is tuned out of the spectrally narrow extended states at the center of the Landau level before the bottom surface is, resulting in a large reduction in the screening of the bottom surface by the top and the breakdown of the quantum Hall effect. For surface states, this effect is exacerbated at total filling factor $\nu=1$, because (i) the filling factor is formed by two halffilled Landau levels and (ii) the importance of the top surface's screening of the bottom surface. By contrast, in a quantum well model with two subbands, the one subband does not screen the other, and the collapse of the quantum Hall effect precisely when the total filling factor $\nu=1$ plateau should appear would be only coincidental.

Measurements that contact the two surfaces separately, which are more challenging and are beyond the scope of this paper, should shed light on the nature of the insulating state [44] and should be especially intriguing at parameters corresponding to $\nu=0$, which does not exist in conventional semiconductor bilayers. Such experiments would also allow us to distinguish explanations for the highly resistive state, such as those described above or electronhole puddles [45].

Finally, it is instructive to contrast the quantum Hall effect of the (001) films with that of (112)-oriented films of similar thickness $[13,14]$. The quantum Hall effect of (112) films is characterized by a degeneracy factor of two that is lifted in fields above approximately $10 \mathrm{~T}$, leading to quantum Hall plateaus of $\nu=1,2,3,4,5$, and 6 [13]. Gated magnetotransport and quantum capacitance reveal ambipolar quantum transport, a zero-energy Landau level, and linear dispersion in the 2D states giving rise to the quantum Hall effect [14]. Here, by contrast, states are nondegenerate, and integer filling factors of $\nu=2$ to $\nu=10$ are due to crossing Landau levels. In the (112)-plane films, the Landau levels do not cross and rather exhibit a "conventional" fan diagram [14]. Moreover, in the (112)plane films, $\nu=1$ and a holelike $\nu=-1$ filling factor are all readily observed [14], in sharp contrast to what is observed here. The salient difference is that (112) surfaces 
are thought to host double Fermi arcs, which can give rise to two Fermi pockets near the center of the surface Brillouin zone $[10,46]$ and, thus, an even integer Hall effect until their degeneracy is lifted by the magnetic field. In other words, a single (112) surface can give rise to an integer quantum Hall effect with the observed characteristics. In contrast, (001) surfaces require the participation of both surfaces to obtain an integer Hall effect [31]. Although we do not know why the bottom surface does not appear to contribute in an obvious way to the quantum Hall effect of (112) films, this result also provides a natural explanation for the absence of the intersurface effects, whether through Coulomb interactions or screening, allowing the development of plateaus at $\nu=-1$ and $\nu=1$.

\section{CONCLUSIONS}

There are two main results of this work. First, by studying the quantum Hall effect in relatively thick, (001)-oriented $\mathrm{Cd}_{3} \mathrm{As}_{2}$ films, we discover a 2D Dirac surface state akin to that of a 3D topological insulator. Besides opening a new direction in the study of 3D topological insulators, based on the realization of highmobility, surface-only transport, this result demonstrates the wide tunability of the 3D Dirac semimetal thin film platform. After all, by contrast, in a very thick slab, one expects the 3D Dirac semimetal to be gapless with surface states depending on microscopic details of the bulk and surface disorder; here, we find a 3D topological insulator. These states are expected to give rise to a quantum spin Hall insulator state in ultrathin films $[8,47]$. The contrast between these films and the more widely studied (112)oriented films discussed above, is equally striking. The second main result of this work is the discovery of a highly insulating state that develops instead of a quantum Hall plateau with filling factor $\nu=1$. This state has not been previously observed in the quantum Hall regime of surface states of 3D TIs. Though we cannot unequivocally identify the nature of this state, it does require an explanation that goes beyond the noninteracting, single-particle description that has successfully described the high-field transport of 3D TIs to date. Intersurface Coulomb interactions seem likely to be responsible, and we speculate that the state may be an excitonic condensate or a breakdown in screening due to the half filling on each surface. While this state is an obvious object of future study, we remark that intersurface interactions represent a promising pathway toward realizing interacting topological systems, a subject of wide general interest.

\section{ACKNOWLEDGMENTS}

The authors thank Allan H. MacDonald, Ross McDonald, and Boris Shklovskii for discussions and the Vannevar Bush Faculty Fellowship program of the U.S. Department of Defense (Grant No. N00014-16-1-2814) for support. Some of the research reported here made use of shared facilities of the UCSB MRSEC (NSF DMR 1720256). A portion of this work was performed at the National High Magnetic Field Laboratory, which is supported by National Science Foundation Cooperative Agreement No. DMR-1644779 and the State of Florida.

[1] C. L. Kane, in Topological Insulators, edited by M. Franz and L. Molenkamp (Elsevier, Amsterdam, 2013), Vol. 6, Chap. 1, pp. 3-34.

[2] M. Z. Hasan and C.L. Kane, Colloquium: Topological Insulators, Rev. Mod. Phys. 82, 3045 (2010).

[3] X. L. Qi and S. C. Zhang, Topological Insulators and Superconductors, Rev. Mod. Phys. 83, 1057 (2011).

[4] N. P. Armitage, E. J. Mele, and A. Vishwanath, Weyl and Dirac Semimetals in Three-Dimensional Solids, Rev. Mod. Phys. 90, 015001 (2018).

[5] X. G. Wan, A. M. Turner, A. Vishwanath, and S. Y. Savrasov, Topological Semimetal and Fermi-Arc Surface States in the Electronic Structure of Pyrochlore Iridates, Phys. Rev. B 83, 205101 (2011).

[6] A. C. Potter, I. Kimchi, and A. Vishwanath, Quantum Oscillations from Surface Fermi Arcs in Weyl and Dirac Semimetals, Nat. Commun. 5, 5161 (2014).

[7] P. J. W. Moll, N. L. Nair, T. Helm, A. C. Potter, I. Kimchi, A. Vishwanath, and J. G. Analytis, Transport Evidence for Fermi-Arc-Mediated Chirality Transfer in the Dirac Semimetal $\mathrm{Cd}_{3} \mathrm{As}_{2}$, Nature (London) 535, 266 (2016).

[8] Z. J. Wang, H. M. Weng, Q. S. Wu, X. Dai, and Z. Fang, Three-Dimensional Dirac Semimetal and Quantum Transport in $\mathrm{Cd}_{3} \mathrm{As}_{2}$, Phys. Rev. B 88, 125427 (2013).

[9] B.-J. Yang and N. Nagaosa, Classification of Stable ThreeDimensional Dirac Semimetals with Nontrivial Topology, Nat. Commun. 5, 4898 (2014).

[10] M. Kargarian, Y. M. Lu, and M. Randeria, Deformation and Stability of Surface States in Dirac Semimetals, Phys. Rev. B 97, 165129 (2018).

[11] B. A. Volkov and O. A. Pankratov, Two-Dimensional Massless Electrons in an Inverted Contact, JETP Lett. 42, 178 (1985), http://www.jetpletters.ac.ru/ps/1420/article_21570 .shtml.

[12] O. A. Pankratov, S. V. Pakhomov, and B. A. Volkov, Supersymmetry in Heterojunctions: Band-Inverting Contact on the Basis of $\mathrm{Pb}_{1-x} \mathrm{Sn}_{x} \mathrm{Te}$ and $\mathrm{Hg}_{1-x} \mathrm{Cd}_{x} \mathrm{Te}$, Solid State Commun. 61, 93 (1987).

[13] T. Schumann, L. Galletti, D. A. Kealhofer, H. Kim, M. Goyal, and S. Stemmer, Observation of the Quantum Hall Effect in Confined Films of the Three-Dimensional Dirac Semimetal $\mathrm{Cd}_{3} \mathrm{As}_{2}$, Phys. Rev. Lett. 120, 016801 (2018).

[14] L. Galletti, T. Schumann, O. F. Shoron, M. Goyal, D. A. Kealhofer, H. Kim, and S. Stemmer, Two-Dimensional Dirac Fermions in Thin Films of $\mathrm{Cd}_{3} \mathrm{As}_{2}$, Phys. Rev. B 97, 115132 (2018).

[15] M. Uchida, Y. Nakazawa, S. Nishihaya, K. Akiba, M. Kriener, Y. Kozuka, A. Miyake, Y. Taguchi, M. Tokunaga, N. Nagaosa, Y. Tokura, and M. Kawasaki, Quantum Hall 
States Observed in Thin Films of Dirac Semimetal $\mathrm{Cd}_{3} \mathrm{As}_{2}$, Nat. Commun. 8, 2274 (2017).

[16] W. Witczak-Krempa, G. Chen, Y. B. Kim, and L. Balents, Correlated Quantum Phenomena in the Strong SpinOrbit Regime, Annu. Rev. Condens. Matter Phys. 5, 57 (2014).

[17] S. Rachel, Interacting Topological Insulators: A Review, Rep. Prog. Phys. 81, 116501 (2018).

[18] S. Borisenko, Q. Gibson, D. Evtushinsky, V. Zabolotnyy, B. Buchner, and R. J. Cava, Experimental Realization of a Three-Dimensional Dirac Semimetal, Phys. Rev. Lett. 113, 027603 (2014).

[19] S. Jeon, B. B. Zhou, A. Gyenis, B. E. Feldman, I. Kimchi, A. C. Potter, Q. D. Gibson, R. J. Cava, A. Vishwanath, and A. Yazdani, Landau Quantization and Quasiparticle Interference in the Three-Dimensional Dirac Semimetal $\mathrm{Cd}_{3} \mathrm{As}_{2}$, Nat. Mater. 13, 851 (2014).

[20] M. Neupane, S. Y. Xu, R. Sankar, N. Alidoust, G. Bian, C. Liu, I. Belopolski, T. R. Chang, H. T. Jeng, H. Lin, A. Bansil, F. Chou, and M. Z. Hasan, Observation of a ThreeDimensional Topological Dirac Semimetal Phase in HighMobility $\mathrm{Cd}_{3} \mathrm{As}_{2}$, Nat. Commun. 5, 3786 (2014).

[21] Z. K. Liu, J. Jiang, B. Zhou, Z. J. Wang, Y. Zhang, H. M. Weng, D. Prabhakaran, S.-K. Mo, H. Peng, P. Dudin, T. Kim, M. Hoesch, Z. Fang, X. Dai, Z. X. Shen, D. L. Feng, Z. Hussain, and Y. L. Chen, A Stable Three-Dimensional Topological Dirac Semimetal $\mathrm{Cd}_{3} \mathrm{As}_{2}$, Nat. Mater. 13, 677 (2014).

[22] I. Crassee, R. Sankar, W.-L. Lee, A. Akrap, and M. Orlita, $3 D$ Dirac Semimetal $\mathrm{Cd}_{3} \mathrm{As}_{2}$ : A Review of Material Properties, Phys. Rev. Mater. 2, 120302 (2018).

[23] A. M. Conte, O. Pulci, and F. Bechstedt, Electronic and Optical Properties of Topological Semimetal $\mathrm{Cd}_{3} \mathrm{As}_{2}$, Sci. Rep. 7, 45500 (2017).

[24] W. Y. Shan, H.Z. Lu, and S. Q. Shen, Effective Continuous Model for Surface States and Thin Films of ThreeDimensional Topological Insulators, New J. Phys. 12, 043048 (2010).

[25] Y. Zhang, K. He, C. Z. Chang, C. L. Song, L. L. Wang, X. Chen, J. F. Jia, Z. Fang, X. Dai, W. Y. Shan, S. Q. Shen, Q. Niu, X. L. Qi, S. C. Zhang, X. C. Ma, and Q. K. Xue, Crossover of the Three-Dimensional Topological Insulator $\mathrm{Bi}_{2} \mathrm{Se}_{3}$ to the Two-Dimensional Limit, Nat. Phys. 6, 584 (2010).

[26] C. Zhang, A. Narayan, S. Lu, J. Zhang, H. Zhang, Z. Ni, X. Yuan, Y. Liu, J.-H. Park, E. Zhang, W. Wang, S. Liu, L. Cheng, L. Pi, Z. Sheng, S. Sanvito, and F. Xiu, Evolution of Weyl Orbit and Quantum Hall Effect in Dirac Semimetal $\mathrm{Cd}_{3} \mathrm{As}_{2}$, Nat. Commun. 8, 1272 (2017).

[27] D. A. Kealhofer, H. Kim, T. Schumann, M. Goyal, L. Galletti, and S. Stemmer, Basal-Plane Growth of Cadmium Arsenide by Molecular Beam Epitaxy, Phys. Rev. Mater. 3, 031201 (2019).

[28] See Supplemental Material at http://link.aps.org/ supplemental/10.1103/PhysRevX.10.011050 for magnetoresistance data of the 50-nm film, details of the $k \cdot p$ model, a discussion of the bulk gap, information about the surface state model, calculated Landau level spectrum for quantum confined bulk states, details of the carrier density determination from the Hall effect, a consistency check of the Landau level indexing scheme, details of how the activation energies are determined, and x-ray data.

[29] R. Jackiw, Fractional Charge and Zero Modes for Planar Systems in a Magnetic Field, Phys. Rev. D 29, 2375 (1984).

[30] A. M. J. Schakel, Relativistic Quantum Hall Effect, Phys. Rev. D 43, 1428 (1991).

[31] L. Fu and C. L. Kane, Topological Insulators with Inversion Symmetry, Phys. Rev. B 76, 045302 (2007).

[32] Y. Xu, I. Miotkowski, C. Liu, J. F. Tian, H. Nam, N. Alidoust, J. N. Hu, C. K. Shih, M. Z. Hasan, and Y. P. Chen, Observation of Topological Surface State Quantum Hall Effect in an Intrinsic Three-Dimensional Topological Insulator, Nat. Phys. 10, 956 (2014).

[33] L. Fu, C. L. Kane, and E. J. Mele, Topological Insulators in Three Dimensions, Phys. Rev. Lett. 98, 106803 (2007).

[34] J. E. Moore and L. Balents, Topological Invariants of TimeReversal-Invariant Band Structures, Phys. Rev. B 75, 121306(R) (2007).

[35] C. Brüne, C. X. Liu, E. G. Novik, E. M. Hankiewicz, H. Buhmann, Y. L. Chen, X. L. Qi, Z. X. Shen, S. C. Zhang, and L.W. Molenkamp, Quantum Hall Effect from the Topological Surface States of Strained Bulk HgTe, Phys. Rev. Lett. 106, 126803 (2011).

[36] S. Tchoumakov, V. Jouffrey, A. Inhofer, E. Bocquillon, B. Placais, D. Carpentier, and M. O. Goerbig, Volkov-Pankratov States in Topological Heterojunctions, Phys. Rev. B 96, 201302(R) (2017).

[37] J. Bodnar, Band Structure of $\mathrm{Cd}_{3} \mathrm{As}_{2}$ from Shubnikov-de Haas and de Haas-van Alphen effects, in Proceedings of the $3 r$ International Conference on the Physics of Narrow Gap Semiconductors, edited by J. Rauluszkiewicz, M. Gorska, and E. Kaczmarek, (PWN-Polish Scientific Publishers, Warszawa, 1977), pp. 311-316; see also arXiv:1709.05845.

[38] J. Cano, B. Bradlyn, Z. Wang, M. Hirschberger, N. P. Ong, and B.A. Bernevig, Chiral Anomaly Factory: Creating Weyl Fermions with a Magnetic Field, Phys. Rev. B 95, 161306(R) (2017).

[39] A. Narayanan, M. D. Watson, S. F. Blake, N. Bruyant, L. Drigo, Y. L. Chen, D. Prabhakaran, B. Yan, C. Felser, T. Kong, P. C. Canfield, and A. I. Coldea, Linear Magnetoresistance Caused by Mobility Fluctuations in n-Doped $\mathrm{Cd}_{3} \mathrm{As}_{2}$, Phys. Rev. Lett. 114, 117201 (2015).

[40] J. P. Eisenstein and A. H. MacDonald, Bose-Einstein Condensation of Excitons in Bilayer Electron Systems, Nature (London) 432, 691 (2004).

[41] D. Tilahun, B. Lee, E. M. Hankiewicz, and A. H. MacDonald, Quantum Hall Superfluids in Topological Insulator Thin Films, Phys. Rev. Lett. 107, 246401 (2011).

[42] B. Seradjeh, J. E. Moore, and M. Franz, Exciton Condensation and Charge Fractionalization in a Topological Insulator Film, Phys. Rev. Lett. 103, 066402 (2009).

[43] Y. W. Suen, L. W. Engel, M. B. Santos, M. Shayegan, and D. C. Tsui, Observation of a $\nu=1 / 2$ Fractional Quantum Hall State in a Double-Layer Electron System, Phys. Rev. Lett. 68, 1379 (1992). 
[44] M. Kellogg, J. P. Eisenstein, L. N. Pfeiffer, and K. W. West, Vanishing Hall Resistance at High Magnetic Field in a Double-Layer Two-Dimensional Electron System, Phys. Rev. Lett. 93, 036801 (2004).

[45] L. A. Ponomarenko, R. Yang, R. V. Gorbachev, P. Blake, A. S. Mayorov, K. S. Novoselov, M. I. Katsnelson, and A. K. Geim, Density of States and Zero Landau Level Probed through Capacitance of Graphene, Phys. Rev. Lett. 105, 136801 (2010).
[46] M. Kargarian, M. Randeria, and Y. M. Lu, Are the Surface Fermi Arcs in Dirac Semimetals Topologically Protected?, Proc. Natl. Acad. Sci. U.S.A. 113, 8648 (2016).

[47] C.X. Liu, H. Zhang, B. H. Yan, X. L. Qi, T. Frauenheim, X. Dai, Z. Fang, and S. C. Zhang, Oscillatory Crossover from Two-Dimensional to Three-Dimensional Topological Insulators, Phys. Rev. B 81, 041307(R) (2010). 